\title{
Migraine and the feeling that "the world is in slow motion"
}

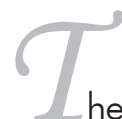

he article by Almeida \& Valença, (1) published in this issue of Headache Medicine, is very curious, describing the case of a woman suffering from migraine, reporting the experience that the "world was in slow motion" on two occasions separated by several years, the first experience having occurred when she was a teenager.

Another patient, a young female, university student, described a similar experience, which occurred during a migraine attack while she was trying to cross a busy road, Caxangá Avenue, in the city of Recife. She had the sensation that vehicles and people were passing "in slow motion". The phenomenon lasted for several minutes. As the patient recognized that something "out of the ordinary" was happening, she did not cross the avenue, thus avoiding being run over by speeding automobiles. Such an illusory sensation can have catastrophic consequences if not readily identified by the individual.

In the movie Matrix, the "bullet time" effect is seen in the trajectory of the bullet moving in slow motion towards the actor. Thomas A. Anderson (nicknamed Neo) is a fictional character in the film. However, in the film, Neo - played by Keanu Reeves - using the "slow motion" phenomenon, was able to divert his body away from the bullets. Our patient probably would not have had that ability.

Humans and certain animals possess the ability to quantify the passage of physical time and perceive the subjective temporal experience of day-to-day activities. ${ }^{(2,3)}$ The perception of the "passing of time" is thus a vital sensory experience in the human species. We know subjectively what seconds, minutes, hours and even days mean, even without the aid of a clock. I consider this function to be of sensory nature, despite the fact that classical books of neurology make no mention of this essential neural function that we use unwittingly throughout our lives.

In addition to this property of the brain in unwittingly quantifying the time in which a given event occurred, there is also the "mental time" perceiving what is present, past, and future..$^{(4)}$

The illusion of time perception is a disturbance of brain function, but since time perception is the result of an interaction between various areas of the brain, changes in this function can be induced in normal individuals through experimental procedures. ${ }^{(2)}$

In an interview conducted by the BBC, the neuroscientist David Eagleman said: "My interest in time and the passage of time came about because when I was eight, I fell from the roof of a house that was being built. In addition, the fall seemed to take a long time."(5) Eagleman even completed his story by saying, "I thought of the book Alice in Wonderland, and what it must have looked like to her when she fell into the rabbit hole."

I report this very curious fact here because a fellow neurologist, years ago, reported during a scientific meeting that he also, when falling from a roof, had the same "slowmotion sensation," but with the perception of being out of his body and seeing the downward movement of his body until he ended up sitting on the ground. He said that at the time there was no cranial trauma or loss of consciousness. 
Such "perceptual distortions of time" can occur when one experiences an extreme situation of imminent risk of death, such as in traffic accidents and muggings. ${ }^{(6)}$

In an experiment, Eagleman studied the possibility of time being perceived differently by subjects under a great emotion in free fall from a structure more than 30 meters high. ${ }^{(2,5)}$ If the perception of time were different, i.e. slower, in the fall condition, they would perceive numbers on a monitor attached to the wrist that passing at different speeds. Under normal conditions, it would be impossible to identify the change in numbers unless the time passed in slow motion for these people. ${ }^{(2,5)}$ The conclusion of the study was that the individual had the illusion that time was dilating, but were unable to identify the correct and expected changes in the numbers in the case of a real time dilatation. Thus, there was no Matrix effect, as in the film cited above, when both the individual and the environment are in another different, but for them identical, dimension of time. Many physicists consider time to be the fourth dimension.

This event is known in neuroscience as the effect of time dilation. (7) Several experiments have demonstrated that it is a physiological cerebral function ${ }^{(8)}$ and, therefore, a disturbance in the physiology of the nervous system can cause a failure in the perception of time. ${ }^{(9)}$

The neurobiological process of how an individual understands time still needs to be further clarified. The encounter of patients with migraine and illusion in the perception of time may shed some light on the understanding of this phenomenon so crucial in the life of human beings.

\section{REFERENCES}

1. Almeida LCA, Valença MM. "A slow-motion world" as a manifestation of Alice in Wonderland syndrome: a case report. Headache Medicine. 2017;8(4):134-137. [Portuguese]

2. Eagleman DM. Human time perception and its illusions. Curr Opin Neurobiol. 2008 Apr; 18(2): 131-6. doi: 10.1016/i.conb.2008.06.002. Epub 2008 Aug 8.

3. Durstewitz D. Neural representation of interval time. Neuroreport. 2004 Apr 9; 15(5):745-9.

4. Kitazawa S. [The science of the mental present: implications of temporal illusions]. Brain Nerve. 2013 Aug;65(8):91 1-21. [Article in Japanese]

5. Neurocientista explica efeito "câmara lenta" relatado por pessoas em situações extremas. https://zap.aeiou.pt/neurocientista-explica-efeitocamera-lenta-relatado-pessoas-viveram-situacoes-extremas-168591 Por ZAP - 31 Julho, 2017

6. Stetson C, Fiesta MP, Eagleman DM. Does time really slow down during a frightening event? PLoS One. 2007 Dec 12;2(12):e1295.

7. van Wassenhove V, Wittmann M, Craig AD, Paulus MP. Psychological and neural mechanisms of subjective time dilation. Front Neurosci. 2011 Apr 26;5:56. doi: 10.3389/fnins.2011.00056. eCollection 2011.

8. Sucala M, David D. Slowing down the clock: a review of experimental studies investigating psychological time dilation. J Gen Psychol. 2012 Oct-Dec;139(4):230-43. doi: 10.1080/00221309.2012.695410.

9. Eagleman DM, Tse PU, Buonomano D, Janssen P, Nobre AC, Holcombe AO. Time and the brain: how subjective time relates to neural time. J Neurosci. 2005 Nov 9;25(45):10369-71.

Marcelo Moraes Valença Full Professor, Neurology and Neurosurgery, Federal University of Pernambuco, Recife, Pernambuco, Brazil. mmvalenca@yahoo.com.br 


\section{Cefaleia e o fenômeno da sensação de que "o mundo está em câmera lenta"}

artigo de Almeida \& Valença, (1) publicado nesta edição da Headache Medicine, é por demais curioso, descrevendo o caso de uma mulher, que sofre de migrânea, relatando a experiência de que o "mundo estava em câmera lenta" em duas ocasiões separadas por vários anos, a primeira experiência ainda quando era adolescente.

Relato semelhante foi descrito por outra paciente, uma jovem mulher, universitária, que durante uma crise de migrânea, ao tentar atravessar uma via muito movimentada na cidade de Recife, a avenida Caxangá, percebeu que os veículos e as pessoas passavam "em câmera lenta". O fenômeno permaneceu por vários minutos. Como a paciente mantinha o discernimento de que algo estava "fora do esperado", não atravessou a avenida, evitando assim de ser atropelada pelos automóveis que passavam com certa rapidez. Essa sensação ilusória pode ter consequências catastróficas se não identificada prontamente pelo indivíduo.

No filme "Matrix", o efeito bullet time é visto no movimento da bala em câmera lenta em direção ao ator. Thomas A. Anderson (apelidado como Neo) é um personagem fictício no filme. No entanto, no filme, Neo - interpretado por Keanu Reeves utilizando o fenômeno "câmera lenta", é capaz de desviar seu corpo das balas. A nossa paciente provavelmente não teria tal habilidade.

Humanos e certos animais possuem a capacidade de quantificar a passagem do tempo físico e percebem a experiência subjetiva do intervalo de tempo durante as atividades do dia a dia..$^{(2,3)} \mathrm{A}$ percepção do "passar do tempo" é, portanto, uma experiência sensorial vital na espécie humana. Sabemos subjetivamente o que significam segundos, minutos, horas e até dias, mesmo sem o auxílio de um relógio.

Eu considero essa função como da ordem sensorial, apesar de os livros clássicos da Neurologia nada falarem dessa função neural de extrema importância que usamos continuamente ao longo da vida. Ilusão da percepção do tempo é um distúrbio da função cerebral, porém, como a percepção de tempo é o resultado de uma interação entre várias áreas cerebrais, alterações desta função podem ser induzidas em indivíduos normais através de procedimentos experimentais. ${ }^{(2)}$

Além dessa propriedade do encéfalo de subjetivamente quantificar o período de tempo em que um determinado evento ocorreu, há ainda o tempo mental do que é presente, passado e futuro. ${ }^{(4)}$

Em entrevista à BBC o neurocientista David Eagleman contou: "O meu interesse pelo tempo e pela passagem do tempo surgiu porque, quando tinha oito anos, caí do telhado de uma casa que estava a ser construída. E a queda pareceu demorar muito tempo".(5) Eagleman ainda completou seu relato falando: "Pensei no livro Alice no País das Maravilhas, e em como devia ter sido parecido para ela, quando caiu no buraco do coelho." (5)

Relato esse curiosíssimo fato porque um neurologista amigo nosso, anos atrás, relatou durante uma reunião científica que também ao cair de um telhado teve a mes- 
ma "sensação de câmera lenta", porém com a percepção de estar fora de seu corpo e vendo a queda de seu corpo até cair sentado no solo. Afirmou que, na ocasião, não houve trauma craniano, nem perda da consciência.

Tais "distorções na percepção do tempo" podem ocorrer quando alguém experimenta uma situação extrema de risco iminente de morte, como em um acidente de trânsito ou durante um assalto. ${ }^{(6)}$

Em experimento bem elaborado, Eagleman estudou a possibilidade de o tempo ser percebido de forma diferente em pessoas em queda livre sob grande emoção, de uma estrutura com mais de 30 metros de altura. ${ }^{(2,5)}$ Se a percepção do tempo fosse diferente, i.e. mais devagar, na condição de queda, elas poderiam perceber números em um monitor preso no pulso que passavam com velocidades diferentes. Em condições normais seria impossível identificar a mudança nos números, a não ser que, para essas pessoas, o tempo passasse em câmera lenta..$^{(2,5)} \mathrm{A}$ conclusão do estudo foi que o indivíduo tinha a ilusão que o tempo se dilatava, não houve o efeito Matrix citado acima, ou seja, de a mbos, o indivíduo e o meio ambiente, estarem numa dimensão diferente, porém idêntica, de tempo. O tempo é considerado por muitos da Física como a quarta dimensão.

Esse evento é conhecido na neurociência como efeito da dilatação do tempo, ${ }^{(7)}$ e vários experimentos têm demonstrado ser um evento cerebral, ${ }^{(8)}$ e, portanto, uma fal ha na função do sistema nervoso pode provocar um distúrbio na percepção do tempo, que pode perdurar por minutos. ${ }^{(9)}$

O processo neurobiológico de como um indivíduo entende o tempo ainda precisa ser melhor estudado. $\bigcirc$ encontro de pacientes com migrânea e ilusão na percepção do tempo pode acender alguma luz na compreensão desse fenômeno tão importante na vida dos seres humanos.

\section{REFERÊNCIAS}

1. Imeida LCA, Valença MM. "Um mundo em câmera lenta" como manifestação da Síndrome de Alice no País das Maravilhas. Headache Medicine. 2017;8(4):134-137.

2. Eagleman DM. Human time perception and its illusions. Curr Opin Neurobiol. 2008 Apr;18(2):131-6. doi: 10.1016/ i.conb.2008.06.002. Epub 2008 Aug 8.

3. Durstewitz D. Neural representation of interval time. Neuroreport. 2004 Apr 9; 15(5):745-9.

4. Kitazawa S. [The science of the mental present: implications of temporal illusions]. Brain Nerve. 2013 Aug;65(8):911-21. [Article in Japanese]

5. Neurocientista explica efeito "câmara lenta" relatado por pessoas em situações extremas. https://zap.aeiou.pt/neurocientista-explicaefeito-camera-lenta-relatado-pessoas-viveram-situacoes-extremas-168591 Por ZAP - 31 Julho, 2017

6. Stetson C, Fiesta MP, Eagleman DM. Does time really slow down during a frightening event? PLoS One. 2007 Dec 12;2(12):e1295.

7. van Wassenhove V, Wittmann M, Craig AD, Paulus MP. Psychological and neural mechanisms of subjective time dilation. Front Neurosci. 2011 Apr 26;5:56. doi: 10.3389/fnins.2011.00056. eCollection 2011.

8. Sucala M, David D. Slowing down the clock: a review of experimental studies investigating psychological time dilation. J Gen Psychol. 2012 Oct-Dec;139(4):230-43. doi: 10.1080/00221309.2012.695410.

9. Eagleman DM, Tse PU, Buonomano D, Janssen P, Nobre AC, Holcombe AO. Time and the brain: how subjective time relates to neural time. J Neurosci. 2005 Nov 9;25(45):10369-71.

Marcelo M. Valença Professor Titular, Neurologia e Neurocirurgia, Universidade Federal de Pernambuco, Recife, Pernambuco, Brasil. Editor-chefe da revista Headache Medicine mmvalenca@yahoo.com.br 